\title{
Pulmonary Hypertension in A Patient With Systemic Sclerosis
}

\author{
Dodiyi-Manuel S.T (Mbbs, Fwacp) ${ }^{1}$ Ezennaka R.C (MBBS) ${ }^{2}$ \\ (Department Of Internal Medicine, University Of Port Harcourt Teaching Hospital) \\ Department Of Internal Medicine,University Of Port Harcourt Teaching Hospital
}

\begin{abstract}
Background: Systemic sclerosis is a chronic connective tissue disease of unknown aetiology that causes widespread microvascular damage and excessive deposition of collagen in the skin and internal organs. There is variability among patients in terms of involvement of skin and internal organs. The heart is involved in 40$70 \%$ of cases. The male to female ratio is $1: 3$ and the incidence increases with age and peaks at $3^{\text {rd }}$ to $5^{\text {th }}$ decade. We report a case of systemic sclerosis complicated by pulmonary hypertension.

Case Presentation: A 35 year old woman presented with recurrent breathlessness, dry cough and leg swelling of 4 years duration following child birth which were associated with orthopnoea, easy fatigueability, presyncope, weight loss, dysphagia and swollen painful digits. Examination revealed a chronically ill looking woman in respiratory distress with $\mathrm{SPO}_{2}$ of $86 \%$ in room air. Investigations done were suggestive of systemic sclerosis and pulmonary hypertension. She was commenced on intermittent oxygen at $3 \mathrm{~L} / \mathrm{min}$, IV omeprazole 40mg daily, Tabs Sildenafil $25 \mathrm{mg}$ daily and Tabs Allopurinol 100mg daily. She made some clinical improvement and was referred to the rheumatologist.

Conclusion: This case report emphasizes the fact that heart involvement in systemic sclerosis may have serious clinical implications. The role of echocardiography in the diagnosis and management has been well established and the treatment is targeted at the organ or system involved.
\end{abstract}

Keywords: Systemic sclerosis, pulmonary hypertension, interstitial lung fibrosis.

\section{Introduction}

Systemic sclerosis is a chronic connective tissue disease of unknown aetiology that causes widespread microvascular damage and excessive deposition of collagen in the skin and internal organs ${ }^{1}$. It affects all races and distributed worldwide.There is variability among patients in terms of involvement of skin and internal organs. The heart is involved in $40-70 \%$ of cases ${ }^{2,3}$. Scleroderma heart disease or in combination with interstitial lung fibrosis is one of the main determinants of overall prognosis ${ }^{2}$. Studies have shown that pulmonary hypertension which affects $10-15 \%$ of patients with systemic sclerosis ultimately causes right ventricular failure and death.It is a rare disease relatively, with an incidence of 2-20 per million persons annually. Factors such as age, sex, genetic background and environmental exposure may influence susceptibility. The male to female ratio is $1: 3$ and the incidence increases with age and peaks at $3^{\text {rd }}$ to $5^{\text {th }}$ decade $^{5}$. We report a case of systemic sclerosis complicated by pulmonary hypertension.

\section{Casepresentation}

A 35year old woman presented with recurrent breathlessness, dry cough and leg swelling of 4 years duration following child birth. These are said to have worsened two weeks prior to presentation. She also had orthopnoea, easy fatigueability, presyncope weight loss,dysphagia and swollen painful digits. No fever, vomiting,or menorrhagia.She was neither hypertensive nor diabetic. She had received anti tuberculous medications for 18months, prior to presentation although genexpert and Acid fast bacilli were negative. She had been managed at various health facilities for heart failure in the past. Physical examination revealed a chronically ill looking woman in respiratory distress with $\mathrm{SPO}_{2}$ of $86 \%$ in room air. She had reduced breath sounds with basal coarse lung crepitations, hepatomegaly and edematous cold extremities. There were swollen digits with hardening and loss of the right index finger tip and hyperpigmentation over the anterior chest wall.

Electrocardiogram (ECG) done on presentation showed low QRS voltages, sinus tachycardia, right axis deviation and ischemic changes in inferior and anterolateral leads. Echocardiography revealed dyskinesia of the interventricular septum, with ejection fraction(EF) of $50.2 \%$, dilated right atrium, thickened right ventricular walls, poor right ventricular systolic function, severe tricuspid regurgitation with pulmonary hypertension (right ventricular systolic pressure of $56 \mathrm{mmHg}$ ). There was fibrosis of the aortic and mitral valves with thickened pericardium, pericardial and pleural effusion. An earlier chest radiograph showed widespread alveolar shadowing in both lung fields. Serology done was positive for anti-nuclear factor (ANF), and extractable nuclear antigen antibodies (SCL-70 AB). 
She was commenced on intermittent oxygen at $3 \mathrm{~L} / \mathrm{min}$, IV omeprazole $40 \mathrm{mg}$ daily, Tabs Sildenafil 25mg daily and Tabs Allopurinol 100mg daily. She made some clinical improvement and was referred to the rheumatologist.

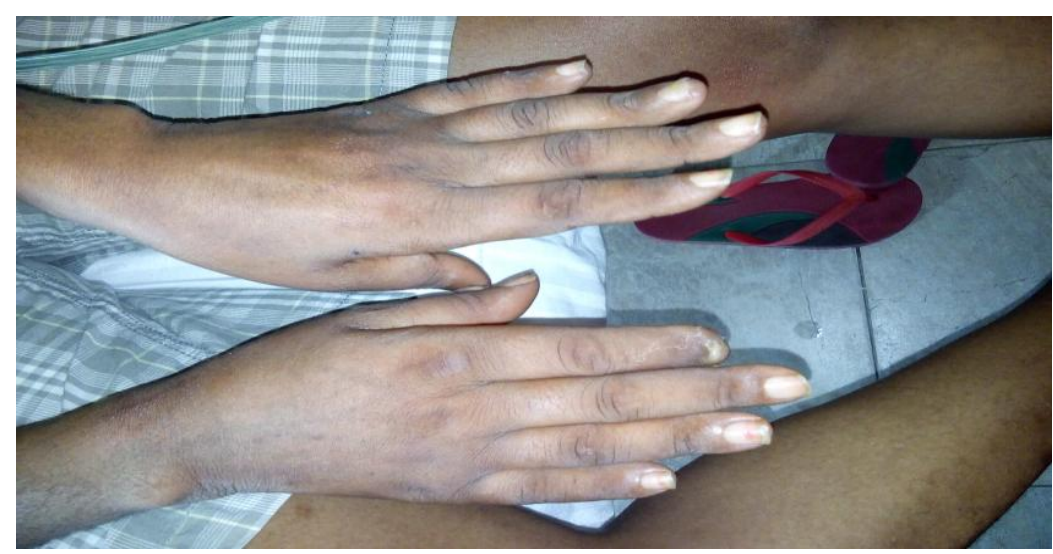

Figure 1

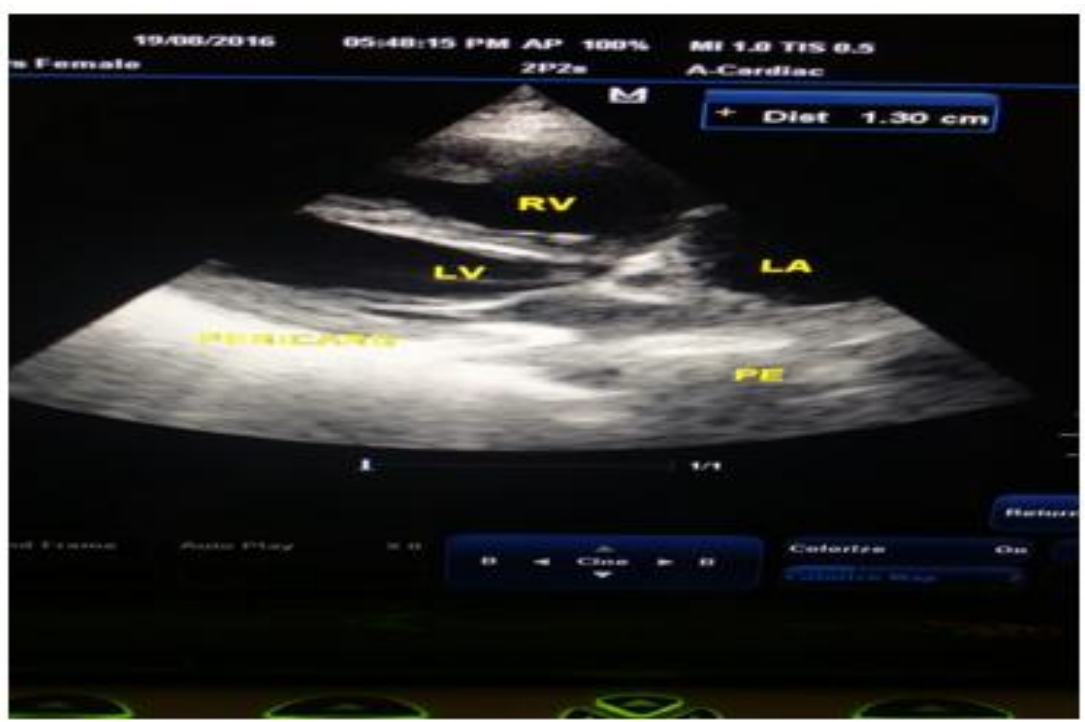

Figure 2

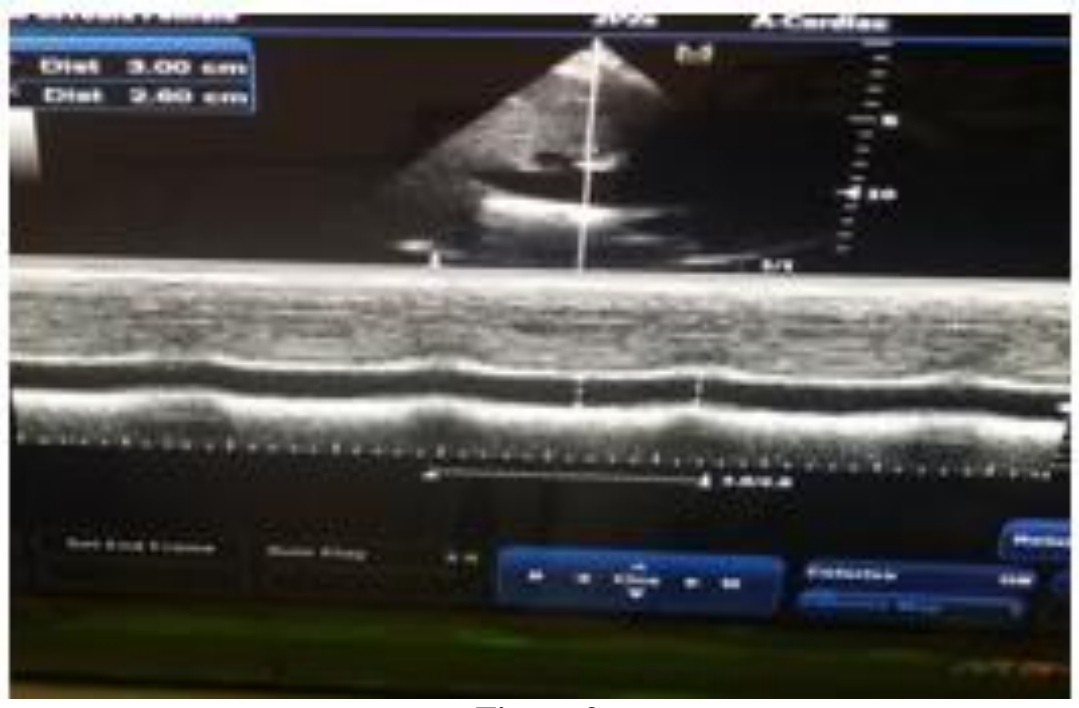

Figure 3 


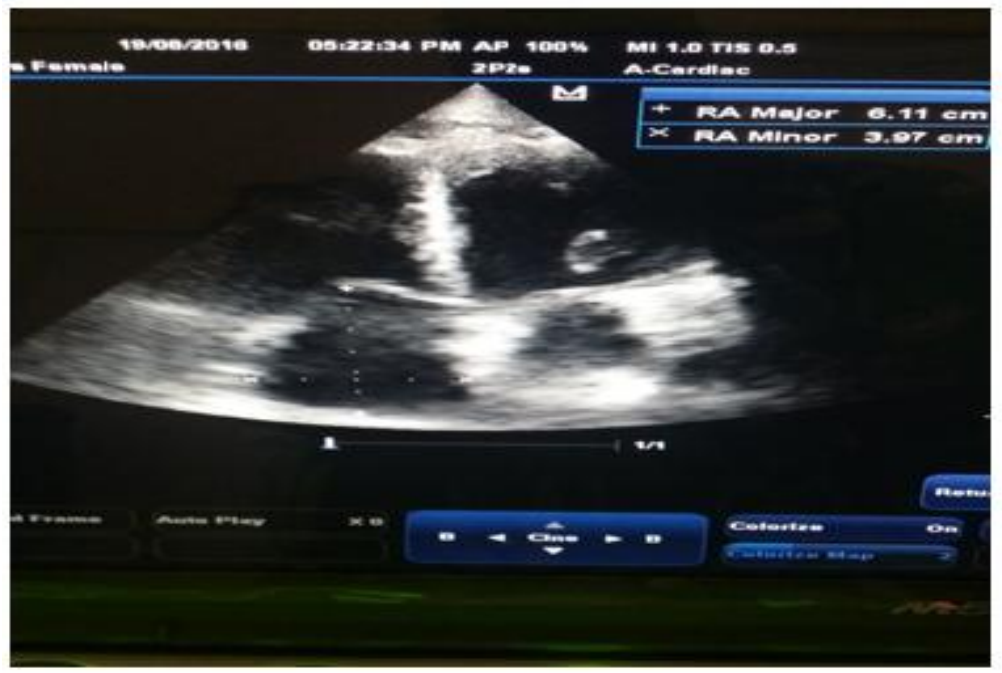

Figure 4

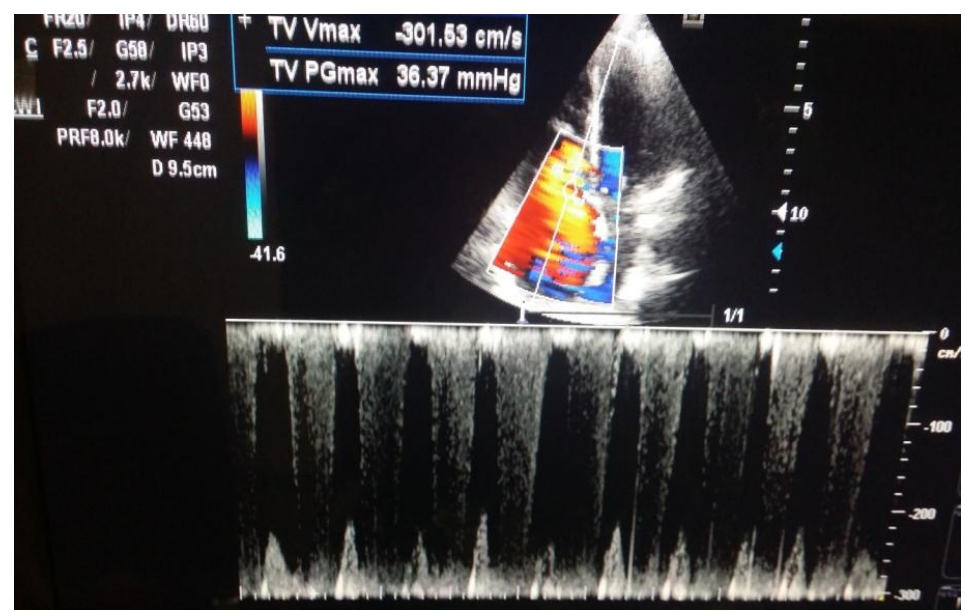

Figure 5

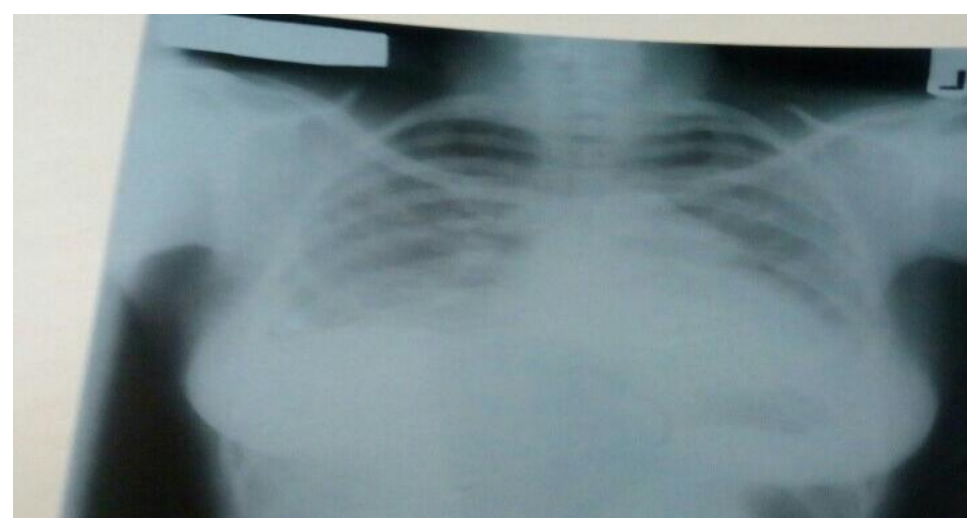

Figure 6

\section{Discussion}

There is a variation of systemic sclerosis which ranges from generalized cutaneous thickening to a form distinguished by restricted skin involvement (mostly face and fingers) and slow progression. It usually takes several decades before full manifestation of characteristic internal involvement. This is known as limited cutaneous scleroderma or CREST syndrome (Calcinosis Cutis, Raynaud's Phenomenon, Oesophageal Dysfunction, Sclerodactyly and Telengiectasia). The commonest initial clinical manifestation is Raynaud's phenomenon identified by pallor, cyanosis and/or rubor of the distal portions of the digits after exposure to cold $^{7}$. Occasionally, symptoms like heartburn, dysphagia or dyspnea are the first manifestations of the disease ${ }^{6}$.Oesophageal dysfunction is the commonest gastro intestinal manifestation. Dysphagia initially results 
from impaired oesophageal motility but may also be caused by gastrooesophageal reflux disease and secondary strictures. Barrett's oesophagus occurs in one-third of patients with scleroderma and these patients have an increased predisposition to complications like stricture and adenocarcinoma ${ }^{6,8}$.

Interstitial lung disease, pulmonary hypertension, pleuritis, pleural effusion and aspiration pneumonia are the pulmonary manifestations of systemic sclerosis ${ }^{9}$. The involvement of the lung varies among individuals. There is a possibility of lung involvement when a patient with systemic sclerosis presents with dyspnea and dry cough. Nevertheless, chronic cough may be the only sign of pulmonary disease in systemic sclerosis ${ }^{6,7}$. Pulmonary hypertension which may result from longstanding interstitial and peri-bronchial fibrosis or intimal hyperplasia of small pulmonary arteries is more commonly seen in limited sclerosis than with the diffuse disease. It is often a late presentation.

There may be signs of right sided heart failure (elevated jugular venous pressure, pitting pedal oedema, right ventricular heave) and accentuation of second heart sound (S2) on physical examination ${ }^{7,8}$. The diagnosis is confirmed by echocardiogram or right sided cardiac catheterization ${ }^{7,8}$. When compared to the general population, hypertension and atherosclerosis occur at a similar or increased rates ${ }^{10}$. Pericardial effusion (with pericarditis), constrictive pericarditis, arrhythmias and congestive cardiac failure are the cardiac complications associated with systemic sclerosis. Cardiac failure may result from diffuse fibrous replacement of cardiac muscles, pulmonary hypertension or secondary corpulmonale. It tends to be chronic and difficult to treat ${ }^{6}$. The renal crisis associated with systemic sclerosis presents with an abrupt rise in blood pressure over days to weeks and rapidly progressive renal failure if untreated, usually within the first 5 years of the disease ${ }^{11}$. The presentation ranges from mildly elevated or normal blood pressure to malignant hypertension resulting in elevated plasma renin levels, increased serum creatinine, proteinuria, and microangiopathichaemolytic anaemia ${ }^{7}$.

Criteria to identify patients with systemic sclerosis have been proposed by the American College of Rheumatology and they include either 1 major or 2 minor criteria ${ }^{12}$. Major criteria include scleroderma proximal to the metacarpophalangeal joints and minor criteria are sclerodactyly, digital pitting scars and bibasilar pulmonary fibrosis. Investigationsdone include pulmonary function test, chest $\mathrm{x}$-ray or high resolution computerizedtomography scanfor patients with pulmonary symptoms and electrocardiogram, echocardiogram, stress test and cardiac catheterization for cardiac symptoms. Gastrointestinal endoscopy is done for patients with gastrointestinal symptoms. Patients who complain of Raynaud's phenomenon may have capillary microscopy done $^{7,8}$ In $90 \%$ of cases of systemic sclerosis, antinuclear antibodies have been detected ${ }^{12}$. Autoantibodies to topoisomerase-1 (anti-scl-70) are more associated with diffuse systemic sclerosis while anti-centromere antibodies are associated with limited systemic sclerosis. Nevertheless, the sensitivity of these tests is $\operatorname{low}^{13}$. Treatment is targeted at the organ or system involved. Treatment of skin lesions includes use of ultraviolet light, high dose topical corticosteroid, topical antibiotic ointments and oral analgesics.

\section{Conclusion}

This case report emphasizes the fact that cardiac involvement in systemic sclerosis may have serious clinical implications. The presence of chronic cough and fever even in endemic areas is not always tuberculosis. The role of echocardiography in the diagnosis and management has been well established and the treatment is targeted at the organ or system involved.

\section{Reference}

[1]. Hinchcliff M, VargaJ.Systemic sclerosis/scleroderma: a treatable multisystem disease. AmFam Physician 2008; 78: 961-968

[2]. Ferri C, Emdin M, Giuggioli D, et al. Autonomic dysfunction in systemic sclerosis: time and frequency domain 24 hour heart rate variability analysis. Br J Rheum 1997; 36: 669-676.

[3]. Follansbee WP, Miller TR, Curtiss EI et al. A controlled clinicopathologic study of myocardial fibrosis in systemic sclerosis (scleroderma). J Rheumatol 1990; 656-662.

[4]. Hachulla E, Gressin V, Guillevin L, et al. early detection of pulmonary arterial hypertension in systemic sclerosis. Arthritis Rheum 2005; 52: 3792-3800.

[5]. Lawrence RC, Helmick CG, Arurett FC, et al. estimates of the prevalence of arthritis and selected musculoskeletal disorders in the United States. Arthritis Rheum 1998; 41: 778-799.

[6]. Mark H, Robert B. 1999. The Merk Manual of Diagnosis and Therapy. $17^{\text {th }}$ ed. Merck and co. USA.

[7]. Eisenberg et al. systemic sclerosis. Hospital Physician January, 2008. Pp: 33-38.

[8]. Dennis L Kasper et al. 2005. Harrisons Principles of Internal Medicine. 16 ed. McGraw-Hill USA. Pp 1979-1990.

[9]. Owens GR, Follansbee WP. Cardiopulmonary manifestations of systemic sclerosis. Chest 1987; 91: 118-127.

[10]. Kawai S, Fukuda Y, Okada R. Atherosclerosis of the coronary arteries in collagen disease and allied disorders with special reference to vasculitis as a preceeding lesion of coronary atherosclerosis. JpnCirc J 1982; 11: 1208-1221.

[11]. Steen VD. Scleroderma renal crisis. Rheum Dis Clin North Am 1996; 22: 861-878.

[12]. Subcommittee for the scleroderma criteria for the American Rheumatism Association Diagnostic and Therapeutic Criteria Committee. Preliminary criteria for the classification of systemic sclerosis (scleroderma). Arthritis Rheum 1980; 23: 581-590.

[13]. Bernstein RM, Steigerwald JC, Tan EM. Association of antinuclear and antinucleolar antibodies in progressive systemic sclerosis. ClinExpImmunol 1982; 48: 43-51.

[14]. Kreuter A, Breuckmann F, Uhle A, et al. Low dose UVA 1 phototherapy in systemic sclerosis: effects on acrosclerosis. J Am AcadDermatol 2004; 50: 740-747. 